\title{
Pregnancy with Guillain-Barre Syndrome-Anaesthetic Challenges and Management
}

\section{Mridu Paban Nath*}

Department of Anaesthesiology and Critical Care, Gauhati Medical College Hospital, Guwahati, Assam, India

\begin{abstract}
We report the case of a 27 year old woman who was diagnosed with an axonal type of Guillain-Barré syndrome at 36 weeks' gestation. The patient had severe motor loss but she was treated effectively with physiotherapy and general care in intensive care unit and she underwent cesarean delivery with general anesthesia at 38 weeks and gradual motor power recovered over a period of 3 months.
\end{abstract}

Keywords: Guillain-Barre syndrome; Immunoglobulin; Pregnancy

\section{Introduction}

Guillain-Barré syndrome is an acute inflammatory, demyelinating polyradiculoneuropathy characterized by progressive motor weakness, areflexia, and ascending paralysis. Patients usually have a history of upper respiratory tract infection or gastroenteritis within one to three weeks before the onset of disease. It presents with weakness that first involves the extremities, followed by involvement of the trunk, neck, and facial muscles. In severe cases, loss of reflexes, motor paralysis, and respiratory failure can occur. Other clinical features, such as sensory symptoms, cranial nerve involvement, and autonomic dysfunction, may be present. The reported incidence of this syndrome in the general population is approximately $1.7 / 100,000$ per year [1].

\section{Case Report}

A 27 year old primipara at 36 weeks of pregnancy was admitted at our hospital with recent history of progressive numbness and weakness in all limbs, and inability to walk. The muscle weakness started with the lower extremities and progressed to the upper extremities. Her symptoms developed over a period of 10 days. The patient's recent history and pregnancy until that point been unremarkable. There was no history of difficulty in breathing and swallowing, although she complained of bowel and urinary incontinence.

Physical examination revealed a well-nourished young women who were fully conscious and alert. All vital signs were normal. Neurological examination on admission showed that the muscle strength was 2 (on a 0-5 Medical Research Council [MRC] scale) for lower limb distal muscles and 3 for lower limb proximal, upper limb distal and proximal muscles. She had no knee or ankle reflexes. There was also no sensory deficit or disturbance of cranial nerve function.

Routine laboratory screening tests and full blood counts were all within normal limit. The results of serologic tests including Human Immunodeficiency Virus (HIV) were negative. The patient was admitted at intensive care unit (ICU) with a presumed diagnosis of Guillain- Barre syndrome (GBS), which were supported by CSF examination (protein $131 \mathrm{mg} / \mathrm{dl}$, glucose $3.6 \mathrm{mmol} / \mathrm{L}$ and no cells).

Obstetric examination revealed the height of the uterine fundus corresponding to the stage of gestation with a single fetus presenting cephalically. The liquor volume was normal and the estimated weight of the fetus was approx. $2 \mathrm{~kg}$. The fetal heart beat was present. Vaginal examination revealed a normal gynaecoid pelvis, and the cervix was 2 $\mathrm{cm}$ long with closed internal os.

The patient's neurological condition deteriorated over the next 24 hour and she had progressive worsening of muscle strength
( 1 on a $0-5$ MRC scale) in the upper and lower limb. She showed no signs of respiratory failure requiring ventilator support. She was managed conservatively with regular monitoring of arterial blood gases (ABG) and prophylactic anticoagulation was started to prevent thromboembolism. Intravenous Immunoglbulin (IVIG) and plasmapheresis was not started, as patient could not afford the cost of it. Patient was catheterized and regular physiotherapy and rehabilitation was started in the ICU. Patient was haemodynamically stable and showed no signs of autonomic dysfunction throughout her ICU stay.

On the $5^{\text {th }}$ day of admission, patient developed premature rupture of membrane without any progress of labour. So she was planned for elective caesarean section on the next day. Patient was given Ranitidine tablet $150 \mathrm{mg}$ on the previous night and on the morning of surgery.

In the operating room, an intravenous cannula was inserted \& balanced crystalloid solution was started. Metochlorpramide $10 \mathrm{mg}$ injection I.V. given before induction of anesthesia. Electrocardiogram, Pulse oxymetry and non-invasive blood pressure measurement were attached. Patient was induced with Thiopentone sodium $4 \mathrm{mg} / \mathrm{kg}$ and Atracurium $0.08 \mathrm{mg} / \mathrm{kg}$ and trachea secured with $7.5 \mathrm{~mm}$ size tube. Anesthesia maintained with $\mathrm{O}_{2}: \mathrm{N}_{2} \mathrm{O}(1: 1)$ till delivery of baby. The time from induction to delivery was 9 minutes and the patient delivered healthy $(2.4 \mathrm{~kg}$ and $43 \mathrm{~cm}$ ) with 5 and 10 minutes apgar scores of 7 and 10 minutes respectively. After delivery of the baby, $100 \mu g m$ Fentanyl administered I.V and Isoflurane was started. Patient required an additional dose of top-up muscle relaxant and was haemodynamically stable and showed no signs of autonomic nervous system dysfunction throughout the operation. The last surgical suture was placed 40 minutes after surgical incision and the wound was infiltrated with $0.25 \%$ Bupivacaine $20 \mathrm{ml}$. Patient was reversed after full clinical recovery of the muscle relaxants with Neostigmine $2.5 \mathrm{mg}$ and Glycopyrrolate 0.5 mg.

Postoperatively patient was shifted to the ICU and neurological assessment showed no deterioration from the pre-operative period. Patient was given intermittent Fentanyl \& Ketorolac injection for

${ }^{*}$ Corresponding author: Mridu Paban Nath, Department of Anaesthesiology and Critical Care, Gauhati Medical College Hospital, Guwahati, Assam, India, Tel: 09957630954, 09207308919; E-mail: drmridupaban@yahoo.com

Received April 21, 2014; Accepted August 23, 2014; Published August 25, 2014

Citation: Nath MP (2014) Pregnancy with Guillain-Barre Syndrome-Anaesthetic Challenges and Management. J Clin Case Rep 4: 397. doi:10.4172/21657920.1000397

Copyright: (c) 2014 Nath MP. This is an open-access article distributed under the terms of the Creative Commons Attribution License, which permits unrestricted use, distribution, and reproduction in any medium, provided the original author and source are credited. 
postoperative analgesia. There was no indication of worsening clinical situation or relapse of neurologic symptoms, and the patient was safely discharged on $7^{\text {th }}$ postoperative day. On the day of discharge, the neurological examination revealed improvement of muscle power to ( 2 on a $0-5$ MRC scale). After 3 months of the caesarean delivery, the patient gradually improved her symptoms and able to walk without any aid \& she showed no muscle weakness of the upper or lower extremities.

\section{Discussion}

GBS is an inflammatory demyelinating disease of peripheral nerves, rarely complicates pregnancy. The exact etiology is not established but may represent an aberrant immune response. The occurrence in pregnancy is similar to that in the general population: $1.7 / 100000$ population ${ }^{1}$. Severe GBS is extremely dangerous when present at the time of delivery. High maternal and perinatal mortality rate $(>10 \%)$ is associated with it. Maternal mortality is usually due to respiratory complications and perinatal due to preterm labour and delivery. The management of the gravid patient with GBS doesn't vary a great deal from that in non-pregnant patients with this disease, the mainstay of treatment being supportive care in an ICU. Our patient was also managed intensively with physiotherapy in ICU.

GBS is the most common cause of acute polyradiculitis. Other causes to be differentiated from it by doing specific investigations. CSF analysis shows an elevated protein without an increase in the number leucocytes which is characteristics of GBS as in our case. Electrodiagnostic studies are helpful in the diagnosis and differentiation of varieties of GBS. Electromyography (EMG) and Nerve conduction velocity (NCV) signals are characteristically slowed in GBS [2]. A radiological test helps to exclude GBS from other neurological diseases like Transeverse myelitis and other compressive myelopathy [3]. Typical Magnetic Resonance Imaging (MRI) findings in GBS are nerve root thickening and enhancement surrounding the conus and extending along the cauda equina, resulting from breakdown in the blood brain barrier, which usually prevents enhancement. The most common site of enhancement is considered to be anterior nerve roots, although enhancement of the posterior roots also seen. In our patient MRI and NCV was not done due to technical reasons.

Management ofGBS consists of supportivecareincluding respiratory assistance, hemodynamic and nutritional support, and identification and treatment of nosocomial infections. Because pregnancy itself is also a strong risk factor for thromboembolism, early administration of prophylactic anticoagulation and regular physiotherapy are important $[4,5]$. In our patient also we started with prophylactic anticoagulant and physiotherapy Administration of plasmapheresis and IVIG treatment is effective in preventing progression of neurologic symptoms in GBS [6]. Plasmapheresis has potential risks such as hypotension, fluid overload, pulmonary edema, septicemia, and abnormal clotting profile [4]. Intravenous immunoglobulin treatment has the advantages of a low risk of complications and ease of application [4]. The efficacy of a massive dose of IVIG (100 g/5 days) was confirmed in pregnant patients who developed Guillain-Barré syndrome $[7,8]$. The main limitation of IVIG is the cost. We couldn't use it in our patient due to the cost only.

Normal uterine contractions are maintained and vaginal delivery is possible in the parturient who presents with Guillain-Barré syndrome. Therefore, the presence of this syndrome in pregnancy is not an indication for cesarean delivery [4,9]. Our patient required cesarean delivery at 37 weeks of gestation for premature rupture of membrane with failed progression of labour. Anesthetic management of the parturient diagnosed with Guillain-Barré syndrome depends on the patient's clinical condition at the time of delivery.

There are no established guidelines for anesthetic management of GBS with pregnancy. GBS occurring in pregnancy is associated with an increased need for ventilator support and an increase in maternal mortality $[10,11]$. The key considerations for the management of anesthesia in patients with GBS are the altered function of the autonomic nervous system and the presence of lower motor neuron lesion. Compensatory cardiovascular responses may be absent, resulting in profound hypotension in response to changes in posture, blood loss or positive airway pressure. So in our patient we opted for general anesthesia for caesarean section.

Succinylcholine should not be administered to these patients, as there is a risk of excessive potassium release from the denervated skeletal muscles. Non-depolarizing muscle relaxants with minimal circulatory effects seem to be a better choice. In our patient, we used Atracurium. Feldman [12] reported a parturient who, within one month of recovering from GBS, had a cardiac arrest due to hyperkalemia that occurred shortly after succinylcholine administration for general anesthesia. Nondepolarizing muscle relaxants should be used with caution in patients diagnosed with GBS, because they may result in prolonged neuromuscular block and the need for postoperative ventilation [5].

There are potential risks with regional anesthesia in patients with neurological diseases. Although there have been concerns regarding the possibility of Guillain-Barré syndrome being triggered by regional anesthesia, there is no evidence that epidural anesthesia causes this syndrome. So, we avoided it. Several cases have been reported in which epidural anesthesia was successfully used for labour and cesarean delivery in such patients [5,13-15]. In all the cases, the patients tolerated the epidural and spinal anesthesia and recovered satisfactorily.

GBS is generally self-limited in children and full recovery can usually be expected $[16,17]$. Spontaneous recovery usually occurs 2-3 weeks after onset of the disease, with most patients regaining full muscle strength and deep tendon reflexes. Residual weakness may remain in some patients. In our patient also, slowly the patient recovers the muscle power over a period of 3 months.

It is our opinion that the management of the gravid patient with GBS doesn't differ much from that in non-pregnant patients with this disease. Supportive care in an ICU remains the cornerstone of treatment. Management of airway and respiratory infection, maintaining fluid and electrolyte balance, nutritional support and effective rehabilitation are essential. Although IVIG and Plasmapheresis has an important role in such patient management, spontaneous recovery is also possible with good physiotherapy and general care.

\section{References}

1. Ravn H (1967) The Landry-Guillain-Barré syndrome. A survey and a clinical report of 127 cases. Acta Neurol Scand 43: Suppl 30:1-64.

2. Nadir ZK, Narulkh M (1998) Electro-diagnostic study of 40 cases presenting as Guillain-Barre Syndrome. Pak J Nuerol 4: 50-54.

3. Alkan O, Yildirim T, Tokmak N, Tan M (2009) Spinal MRI findings of guillainbarré syndrome. J Radiol Case Rep 3: 25-28.

4. Chan LY, Tsui MH, Leung TN (2004) Guillain-Barré syndrome in pregnancy. Acta Obstet Gynecol Scand 83: 319-325.

5. Brooks H, Christian AS, May AE (2000) Pregnancy, anaesthesia and Guillain Barré syndrome. Anaesthesia 55: 894-898.

6. Hughes RAC, Swan AV, Cornblath DR, Hartung HP, Bril V(1997) Randomised trial of plasma exchange, intravenous immunoglobulin and combined 
Citation: Nath MP (2014) Pregnancy with Guillain-Barre Syndrome-Anaesthetic Challenges and Management. J Clin Case Rep 4: 397. doi:10.4172/2165-7920.1000397

treatments in Guillain-Barre syndrome. Plasma Exchange/Sandoglobulin Guillain-Barre Syndrome Trial Group. Lancet 349: 225-230.

7. Yamada H, Noro N, Kato EH, Ebina Y, Cho K, et al. (2001) Massive intravenous immunoglobulin treatment in pregnancy complicated by Guillain-Barré Syndrome. Eur J Obstet Gynecol Reprod Biol 97: 101-104.

8. Seoud M, Naboulsi M, Khalil A, Sarouphim P, Azar G, et al. (1999) Landry Guillian-Barre Strohl syndrome in pregnancy: use of high-dose intravenous immunoglobulin. Acta Obstet Gynecol Scand 78: 912-913.

9. Rockel A, Wissel J, Rolfs A (1994) Guillain-Barré syndrome in pregnancy--an indication for caesarian section? J Perinat Med 22: 393-398.

10. Seoud M, Naboulsi M, Khalil A, Sarouphim P, Azar G, et al. (1999) Landry Guillian-Barre Strohl syndrome in pregnancy: use of high-dose intravenous immunoglobulin. Acta Obstet Gynecol Scand 78: 912-913.

11. Berteau P, Morvan J, Bernard AM, Verjut JP, Cléophax JP (1990) The association of acute polyradiculoneuritis, transitory diabetes insipidus and pregnancy. Apropos of a case and review of the literature. J Gynecol Obstet Biol Reprod (Paris) 19: 793-802.
12. Feldman JM (1990) Cardiac arrest after succinylcholine administration in a pregnant patient recovered from Guillain-Barre syndrome. Anesthesiology 72 942-944.

13. Vassiliev DV, Nystrom EU, Leicht CH (2001) Combined spinal and epidura anesthesia for labor and cesarean delivery in a patient with Guillain-Barre syndrome. Reg Anesth Pain Med 26: 174-176.

14. McGrady EM (1987) Management of labour and delivery in a patient with Guillain-Barré syndrome. Anaesthesia 42: 899

15. Alici HA, Cesur M, Erdem AF, Gursac M (2005) Repeated use of epidura anaesthesia for caesarean delivery in a patient with Guillain-Barré syndrome. Int J Obstet Anesth 14: 269-270.

16. Graf WD, Katz JS, Eder DN, Smith AJ, Chun MR (1999) Outcome in severe pediatric Guillain-Barré syndrome after immunotherapy or supportive care. Neurology 52: 1494-1497.

17. Lundkvist I, Van Doorn PA, Vermeulen M, Brand A (1993) Spontaneous Recovery from the Guillain-Barré Syndrome Is Associated with Anti-idiotypic Antibodies Recognizing a Cross-Reactive Idiotype on Anti-neuroblastoma Cel Line Antibodies. Clinical Immunology nand Immunopathology 67: 192-198. 